\title{
Editorial
}

\section{MRSA: We Can Overcome, But Who Will Lead the Battle?}

\author{
Margreet C. Vos, MD, PhD; Henri A. Verbrugh, $\mathrm{MD}, \mathrm{PhD}$
}

During the past two decades, methicillin-resistant Staphylococcus aureus (MRSA) has become the most prevalent and important antimicrobial-resistant pathogen, causing serious nosocomial and community-acquired infections. This trend continues unabated today, now involving additional classes of antimicrobial agents such as glycopeptides. ${ }^{1}$ The emergence of MRSA is primarily due to the successful spread of a limited number of clones of $S$. aureus representing the most prevalent lineages within its population structure $^{2}$ that have acquired one of the resistance-encoding SSCmec elements. Hospitals and other healthcare institutions have been the primary sites where such strains are transmitted and are "breeding" sites where MRSA can maintain its highest reproductive rate. High rates of transmission are the consequence of patients' increased susceptibility to acquisition combined with crowding and high rates of contact with healthcare workers (HCWs), who are considered important vectors in the chain of MRSA transmission. The continuing spread of MRSA indicates that recommended preventive strategies have been either inadequate or improperly implemented. For years, MRSA-positive patients and HCWs have been cycled back into the community, where transmission continues, albeit at a lower rate.

In the late 1950 s and early 1960 s, much attention was focused on elucidating the determinants of $S$. aureus carriage, dispersion, and transmission. ${ }^{3}$ We can learn from that experience that transmission will occur within households or other close communities and that colonization of patients will not end spontaneously in all patients. In 1964, Noble et al. found that acquisition of new, resistant strains was enhanced by the use of antibiotics and that nosocomially acquired strains were detected again at readmission after more than 150 days. ${ }^{4}$ Hare and Thomas, in 1956, published an article on the routes of $S$. aureus transmission. Three routes were implicated: (1) transport of $S$. aureus by hands, handkerchiefs, clothing, bedding, or any other object coming into contact with the nose or skin of infected individuals; (2) the release of $S$, aureus into the atmosphere as a result of friction and dislodgment of dried particles from the skin and hair; and (3) transport of "infected particles" by air currents to other individuals. Of particular note, droplet transmission was not found to be a significant route of transmission. ${ }^{5}$ From a follow-up study, they learned that $S$. aureus carriers can contaminate their clothes and surroundings and that most have enough $S$. aureus cells on various sites of their skin and clothing to render them capable of acting as donors of infection. ${ }^{6}$ Antibiotic pressure may act as an enhancing factor for transmission of resistant S. aureus, as was shown in 1964 by Ehrenkranz. ${ }^{7}$ From these 40- to 50-year-old studies, one can conclude that multiple reservoirs and routes of transmission exist when considering the epidemiology of $S$. aureus, regardless of whether they are resistant to antibiotics.

Currently, we are confronted with many unidentified and untreated sources of MRSA, including asymptomatic carriers who are spreading MRSA into the community, possibly to (future) patients and to HCWs. When those individuals are (re)admitted or return to work in a healthcare setting, their MRSA carriage is usually unrecognized and they may thus contribute inadvertently to the nosocomial spread of MRSA.

In regions where MRSA is highly endemic, it is difficult to determine exactly when, where, and to whom transmission occurs. By contrast, in areas where the prevalence of MRSA is kept low, it is possible to determine all patients and HCWs who have been in contact with an identified MRSA carrier and to test all contacts for MRSA carriage. This search for contacts together with isolation and treatment of carriers are cornerstones of the Dutch "search and destroy" policy that has been so successful. ${ }^{8,9}$ An important component of this approach is having a highly sensitive screen for detecting secondary carriers (ie, one should apply a broad definition of what constitutes a MRSA contact). In a hospital, in addition to roommates, all HCWs and patients on a ward who have had direct or indirect contact with a MRSA carrier are at risk for transmission; they all are at risk of acquiring MRSA. Using a sensitive culture method is likewise important for maintaining a highly sensitive screen for MRSA; this usually requires use of a selective enrichment broth. ${ }^{10}$ The method of isolation chosen should exploit the knowledge gained during the past 50 years regarding the epidemiologic behavior of $S$. aureus. This means that patients should be placed in a separate

Dr. Vos and Professor Dr. Verbrugh are from the Department of Medical Microbiology and Infectious Diseases, Erasmus MC University Medical Center, Rotterdam, the Netherlands.

Address reprint requests to Margreet $C$. Vos, MD, PhD, Dr Molewaterplein 40, 3015 GD Rotterdam, the Netherlands. m.vos@erasmusmc.nl 
room with the door closed, and that HCWs should wear full protection garments (gown, gloves, and mask) when entering that room. Treatment of carriers to prevent further transmission should be instigated as soon as the chance of re-colonization has become low (ie, once wounds, skin lesions, or catheters in situ have healed or been removed, respectively). This policy of search and destroy has been the only one associated with effective MRSA control nationwide, and is probably the key to designing strategies that would decrease MRSA incidence in endemic areas. ${ }^{11,12}$

This issue of Infection Control and Hospital Epidemiology contains five important articles from five different countries on different forms of MRSA surveillance and control. ${ }^{13-17}$ Many research groups are concerned about increasing MRSA infections and are attempting to determine the magnitude of their MRSA problem following publication in 2003 of a Society for Healthcare Epidemiology of America (SHEA) guideline on control of nosocomial MRSA and vancomycin-resistant Enterococcus (VRE) infections. ${ }^{18}$

In the first article in this issue, Lucet et al..$^{13}$ determined the prevalence of MRSA at hospital admission in a 1,100-bed teaching hospital in Paris, France. In an earlier study, they had measured the prevalence of MRSA carriage on admission to the intensive care unit (ICU) and found that screening for MRSA on ICU admission saved money by preventing MRSA infections and their excess costs. They therefore concluded that all patients admitted to the ICU should be screened. ${ }^{19}$ In the current 3-month study, screening cultures of the nose and skin breaks (when present) were performed at hospital admission for patients older than 75 years; 63 (7.9\%) of 797 patients carried MRSA and remained in the hospital for a mean of 15.6 days (983 total patient-days of MRSA carriage). Without this screening, $76 \%$ of the MRSA carriers would have remained unrecognized throughout their entire hospital stay and only 137 (13.9\%) of the MRSA patient-days would have been spent in isolation. More than half of the MRSA carriers had previously been admitted to the same hospital and $16.7 \%$ had been admitted to another hospital within the past 18 months; $13 \%$ were nursing home residents and $5 \%$ were receiving home care at the time of the current admission. Only 4 cases $(6.7 \%)$ had no history of recent healthcare contact. However, the authors did not investigate the possibility of indirect contacts with the healthcare setting (eg, via the patients' household members). Of patients without MRSA, only $33.8 \%$ had been admitted to the same hospital within the past 18 months, a significantly smaller proportion than among the MRSA-positive patients. It seems clear from the epidemiologic data that virtually all MRSA patients in their catchment area had acquired MRSA while in a healthcare facility. Of note, random screening of 9,662 individuals in the population at large recently showed a carriage rate for MRSA of only $0.84 \%$ in the United States. ${ }^{20}$ Random screening of patients being admitted to nonsurgical wards in hospitals in the Netherlands found a rate of $0.03 \%{ }^{21}$ Selective screening of patients on admission on the basis of past medical history with isolation and treatment of MRSA-positive cases therefore seems possible.
Lucet et al. did not actively treat carriers of MRSA. Failure to eradicate such MRSA reservoirs could allow for further nosocomial transmission during the next admission. Detection of MRSA carriers on admission is important to learn how extensively MRSA has infiltrated the healthcare setting and how much the problem has grown. However, an effort to control MRSA should not be limited to screening on hospital admission. A well-integrated (and preferably national) policy would seek to identify, control, and eventually eliminate all reservoirs of MRSA in the healthcare setting (ie, both inpatient and outpatient).

In the second article in this issue, Pan et al. describe an intervention to control nosocomial MRSA in an Italian hospital. ${ }^{14}$ Their hospital had had extremely high rates of MRSA with more than half of all $S$. aureus infections being due to MRSA. Within a cohort of high-risk patient groups, they implemented a MRSA control program by identifying all MRSA-colonized patients through active screening followed by applying simple contact precautions (only routine gloving and handwashing) and eradication therapy (nasal mupirocin three times daily plus chlorhexidine baths once daily, both for 5 days) to all MRSA-positive patients. Incidence rates of MRSA or methicillin-susceptible $S$. aureus (MSSA) bloodstream infection (BSI) were compared during the intervention and preintervention periods. Compared with the preintervention period, the incidence rate of MRSA BSIs was significantly reduced from 0.64 to 0.30 per 1,000 admissions during the intervention period. The impact was greatest in the ICU, which had an $89 \%$ reduction. Methicillin resistance among $S$. aureus blood isolates decreased significantly from $46 \%$ to $17 \%$. It seems very likely that the decrease in MRSA BSIs was due to the active screening, isolation, and treatment program, as there was no decline in nosocomial MSSA BSIs (ie, the decrease was not due to concomitant implementation of some other nonspecific measure that decreased all BSIs). The authors actively treated all MRSA-colonized patients immediately after detection, thereby eliminating many reservoirs of MRSA from the hospital. Some may believe that the simple contact precautions used by Pan et al. played only a minor role in this successful intervention; if so, they should review the results of a similar intervention by Thompson et al. in which eradication of colonization was not attempted. ${ }^{22}$ After 3 years of continually increasing spread of a single strain, MRSA had come to account for almost half of all $S$. aureus infections in that hospital. At that point, surveillance cultures and isolation of all colonized patients began being used and after 1.5 years there were no MRSA infections or colonizations detectable in the hospital despite continuing surveillance cultures. Patients merely colonized were isolated and HCWs were advised to use very good soap and water handwashing after contact with them. The similarity between the two interventions suggests that surveillance cultures and the simple form of isolation used by Pan et al. may indeed have been having an effect. Importantly, albeit quite effective, the intervention by Pan et al. did not eliminate MRSA from the Cremona hospital, indicating that one or more MRSA reservoirs were not completely removed. It 
is possible that this was due to far more than 3 years of spread at the Italian hospital with the development of reservoirs in many, if not all, surrounding acute and longterm-care facilities. Remaining reservoirs may also have included patients colonized but not yet recognized during their first or subsequent admissions. Also, as mentioned by the authors, it is possible that more thorough contact precautions would have been more effective. In a hospital that started off so full of MRSA, it also seems possible that some of the continuing problem could relate to some other reservoir for spread such as colonized HCWs. As long as there are significant numbers of colonized individuals within a hospital, there also may be contamination of the hospital environment that in turn could contribute to spread.

In the third article in this issue of Infection Control and Hospital Epidemiology, Poutanen et al. compared the rate of nosocomial MRSA before, during, and after implementing universal barrier precautions in four hospitals in Toronto, Ontario, Canada, because of the outbreak of severe acute respiratory syndrome (SARS).${ }^{15} \mathrm{~A}$ program of active MRSA surveillance screening of high-risk patients and contact precautions for all colonized patients was already in place in all four hospitals. Extra infection control measures because of the ongoing SARS outbreak were mandated by official directive for 1 month (during parts of April and May 2003) and consisted of using personal protective equipment for all patient contacts (SARS or no SARS) and replacing personal protective equipment between patient contacts. The investigators could not detect a significant decrease in the rate of nosocomial MRSA acquisition as a consequence of these extra infection control measures when compared with the 14-month period prior to this intervention nor when compared with the $6-$ month period following it. Although the authors claim that in three hospitals the incidence rates were somewhat lower after, compared with before, the intervention, their analysis was based on cumulative average rates per period and did not include a more robust analysis of trends. What can we learn from this? Either that the universal application of personal protective equipment for all patients does not constitute an efficient barrier to prevent transmission of MRSA or that compliance rates were too low. Compliance is one of the cornerstones of infection prevention, ${ }^{23}$ but compliance was not directly studied by Poutanen et al. Although the authors question the level of compliance with the personal protective equipment requirement during the SARS outbreak, compliance rates regarding the application of personal protective equipment and other infection control measures in their hospitals were likely higher than those usually observed in healthcare settings not confronted by a SARS threat. This suggests that enhancing adherence to standard precautions alone would not significantly curb the spread of MRSA in the healthcare setting.

What do the infectious diseases experts think of MRSA and its control? Are preventive measures primarily regarded as a nuisance, interfering with delivery of care, or are they regarded as an integral part of protecting those not colonized by MRSA (ie, the vast majority of patients) from such hazards? Certainly, a positive attitude and cooperation with infection control policies are crucial to success in controlling MRSA. Sunenshine et al. give us insight into the opinions of infectious diseases consultants in U.S. hospitals regarding surveillance cultures and isolation measures for drug-resistant organisms including MRSA. ${ }^{16}$ Infectious diseases physicians are often able to see the dramatic consequences of an infection by multidrug-resistant bacteria such as MRSA. It is thus clear why most infectious diseases physicians strongly supported the use of contact precautions for preventing spread from a patient known to have MRSA to other patients on a ward. It is disappointing to learn that only $50 \%$ of the respondents favored the routine use of surveillance cultures for MRSA. Their attitudes and opinions are in contrast to the article by Pan et al. and many other studies ${ }^{18}$ as well as the experience in Northern European countries $^{8,9,11,21}$ demonstrating a decrease in the prevalence of MRSA infections by identification and isolation. ${ }^{14}$ It is not clear why half of those surveyed remained unaware of the benefits of surveillance cultures except that many of those surveyed admitted that they worked in hospitals where surveillance cultures were not being tried much less used regularly like in the Netherlands. Mathematically, it makes little sense to insist on contact precautions only for the occasional patient detected by routine clinical cultures because the vast majority of colonized (and contagious) patients are never detected by routine clinical cultures, as demonstrated in the study of Lucet et al. discussed above. ${ }^{13}$ Active surveillance of patients for MRSA carriage is now recommended by a SHEA guideline ${ }^{18}$; this recommendation was evidence based and classified as category IA. This recommendation seems sound because identifying the MRSA reservoir has clearly been helpful in preventing transmission. ${ }^{18,21}$ All studies of cost-effectiveness to date have concluded that controlling the spread of MRSA costs less than the more expensive infections that occur when it is allowed to spread out of control. $14,18,19,24,25$ The costs of screening and isolation may be further minimized by the recent introduction of novel and rapid assays to detect carriers among patients and HCWs. ${ }^{26,27}$

Finally, "l'histoire se répète."28 The importance of tracking all cases of MRSA is described by Urth et al. in their efforts to control community spread of MRSA. ${ }^{17}$ During a period of 6 years, they meticulously carried out a search and destroy policy in Denmark. They found a particularly virulent clone of leukocidin-positive MRSA, probably of Middle Eastern origin, to spread within certain families of refugees and immigrants and through kindergartens and schools as well as workplaces, thus posing new challenges for infection control. They screened all household members and treated carriers so detected, thereby preventing further spread. They correctly reasoned that if transmission in the community is not prevented, a high prevalence could be reached in the population at large, resulting in introduction of such strains into hospitals. No introduction of that strain into the local hospital was found during the 6 -year study. A robust (inter) national typing system is a prerequisite to be able to track and trace epidemic 
clones of MRSA. Recent studies of the spread of MSSA involved in skin infections have shown that a few strains can be responsible for the majority of all infections..$^{29}$ Those strains have the capability of spreading, partly due to the high density of staphylococci on the exterior of the body during skin infection and partly through other, still unknown, transmission factors. ${ }^{30,31}$ Among collections of both resistant and susceptible isolates of $S$. aureus, epidemic and non-epidemic behavior can be seen, although methicillin-resistant strains are favored in spreading compared with methicillin-sensitive strains. ${ }^{25,30}$ This is also observed when contacts of MRSA carriers are traced and tested. Nevertheless, when a MRSA strain is introduced into a given setting for several days or weeks, spreading is seen in only a few instances. The challenge now lies in unraveling the genes responsible for transmission to be able to focus search and destroy campaigns on strains with a high capacity of spreading (ie, with an inherently high reproductive number). The Danish study is not a signal to throw in the towel and give up. It should be viewed as a stimulant to continue and expand our active search and destroy policies. It is important that new transmission routes and new reservoirs are being studied. New knowledge should be used to improve national policies in a timely fashion as it becomes available so that infection control workers will not fall behind and lose control of MRSA, as many countries have done. The way the Danish actively detect, search, analyze their data, and adjust their methods with MRSA is a good example to policy makers around the world trying to control MRSA in their hospital, region, or country. Although SCCmec type IV strains have been detected in Northern European countries such as Denmark and the Netherlands for the past decade, nosocomial MRSA infection rates remain very low in these countries. Clearly, if we do battle, we will overcome even MRSA. Hospital epidemiologists and infection control professionals should be leading this battle, but in many places they "have not yet begun to fight." In such places, where MRSA infections have been allowed to spread out of control, hospital administrators or politicians may eventually begin to demand better control of this leading hospital pathogen.

\section{REFERENCES}

1. Solberg CO. Spread of Staphylococcus aureus in hospitals: causes and prevention. Scand J Infect Dis 2000;32:587-595.

2. Robinson DA, Enright MC. Multilocus sequence typing and the evolution of methicillin-resistant Staphylococcus aureus. Clin Microbiol Infect 2004:10:92-97.

3. Williams REO. Healthy carriage of Staphylococcus aureus: its prevalence and importance. Bacteriol Rev 1963;27:56-71.

4. Noble WC, Williams REO, Jevons MP, Shooter RA. Some aspects on nasal carriage of staphylococci. J Clin Pathol 1964;17:79-83.

5. Hare R, Thomas CGA. The transmission of Staphylococcus aureus. Br Med J 1956;13:840-844.

6. Hare R, Ridley M. Further studies on the transmission of Staphylococcus aureus. Br Med J 1958;11:69-73.

7. Ehrenkranz NJ. Person-to-person transmission of Staphylococcus aureus. N Engl J Med 1964;271:225-230.

8. Vandenbroucke-Grauls CMJE. Methicillin-resistant Staphylococcus aureus control in hospitals: the Dutch experience. Infect Control Hosp Epidemiol 1996;17:512-513.

9. Verhoef J, Beaujean D, Blok $\mathrm{H}$, et al. A Dutch approach to methicillinresistant Staphylococcus aureus. Eur J Clin Microbiol Infect Dis 1999; 18:461-466.
10. Wertheim H, Verbrugh HA, van Pelt C, de Man P, van Belkum A, Vos MC. Improved detection of methicillin-resistant Staphylococcus aureus using phenyl mannitol broth containing aztreonam and ceftizoxime. $J$ Clin Microbiol 2001;39:2660-2662.

11. Working Party on Infection Prevention. Policy for Methicillin-Resistant Staphylococcus aureus, Guideline 35b. Leiden, the Netherlands: Working Party on Infection Prevention; 2004. Available at www.wip.nl.

12. Meek C. Isolate patients, screen staff to fight MRSA. Canadian Medical Association Journal 2004;171:1158.

13. Lucet J-C, Grenet K, Armand-Lefevre L, et al. High prevalence of carriage of methicillin-resistant Staphylococcus aureus at hospital admission in elderly patients: implications for infection control strategies. Infect Control Hosp Epidemiol 2005;26:121-126.

14. Pan $A$, Carnevale $G$, Catenazzi $P$, et al. Trends in methicillin-resistant Staphylococcus aureus (MRSA) bloodstream infections: effect of the MRSA "search and isolate" strategy in a hospital in Italy with hyperendemic MRSA. Infect Control Hosp Epidemiol 2005;26:127-133.

15. Poutanen S, Vearncombe M, McGeer AJ, Gardam M, Large G, Simor AE. Nosocomial acquisition of methicillin-resistant Staphylococcus aureus during an outbreak of severe acute respiratory syndrome. Infect Control Hosp Epidemiol 2005;26:134-137.

16. Sunenshine RH, Liedtke IA, Fridkin SK, Strausbaugh LJ, The Infectious Diseases Society of America Emerging Infections Network. Management of inpatients colonized or infected with antimicrobial-resistant bacteria in hos pitals in the United States. Infect Control Hosp Epidemiol 2005;26:138-143.

17. Urth T, Juul G, Skov R, Schønheyder HC. Spread of a methicillin-resistant Staphylococcus aureus ST80-IV clone in a Danish Community. Infect Control Hosp Epidemiol 2005;26:144-149.

18. Muto CA, Jernigan JA, Ostrowsky BE, et al. SHEA guideline for preventing nosocomial transmission of multidrug-resistant strains of Staphylococcus aureus and Enterococcus. Infect Control Hosp Epidemiol 2003;24:362-386.

19. Lucet JC, Chevret S, Durand-Zaleski I, Chastang C, Regnier B, Multicenter Study Group. Prevalence and risk factors for carriage of methicillin-resistant Staphylococcus aureus at admission to the intensive care unit: results of a multicenter study. Arch Intern Med 2003;163:181-188.

20. Kuehnert MJ, Hill H, McQuillan G, et al. Prevalence of Staphylococcus aureus colonization in the United States: 2001-2002. Presented at the 42nd Annual Meeting of the Infectious Diseases Society of America; September 30-October 3, 2004; Boston, MA.

21. Wertheim HF, Vos MC, Boelens HA, et al. Low prevalence of methicillinresistant Staphylococcus aureus (MRSA) at hospital admission in the Netherlands: the value of search and destroy and restrictive antibiotic use. J Hosp Infect 2004;56:321-325.

22. Thompson RL, Cabezudo I, Wenzel RP. Epidemiology of nosocomial infections caused by methicillin-resistant Staphylococcus aureus. Ann Intern Med 1982;97:309-317.

23. Pittet D, Hugonnet S, Harbarth S, et al. Effectiveness of a hospital-wide programme to improve compliance with hand hygiene: Infection Control Programme. Lancet 2000;356:1307-1312.

24. Karchmer TB, Durbin LJ, Simonton BM, Farr BM. Cost-effectiveness of active surveillance cultures and contact/droplet precautions for control of methicillin-resistant Staphylococcus aureus. J Hosp Infect 2002;51:126-132.

25. Vriens M, Blok H, Fluit A, Troelstra A, Van Der Werken C, Verhoef J. Costs associated with a strict policy to eradicate methicilin-resistant Staphylococcus aureus in a Dutch university medical center: a 10-year survey. Eur J Clin Microbiol Infect Dis 2002;21:782-786.

26. Huletsky A, Giroux R, Rossbach V, et al. New real-time PCR assay for rapid detection of methicillin-resistant Staphylococcus aureus directly from specimens containing a mixture of staphylococci. J Clin Microbiol 2004;42:1875-1884.

27. Warren DK, Liao RS, Merz LR, Eveland M, Dunne WM Jr. Detection of methicillin-resistant Staphylococcus aureus directly from nasal swab specimens by a real-time PCR assay. J Clin Microbiol 2004;42:5578-5581.

28. Crisostomo MI, Westh $\mathrm{H}$, Tomasz A Chung M, Oliveira DC, de Lencastre H. The evolution of methicillin resistance in Staphylococcus aureus: similarity of genetic backgrounds in historically early methicillin-susceptible and -resistant isolates and contemporary epidemic clones. Proc Natl Acad Sci U S A 2001;98:9865-9870.

29. Koning S, van Belkum A, Snijders S, et al. Severity of nonbullous Staphylococcus aureus impetigo in children is associated with strains harboring genetic markers for exfoliative toxin B, Panton-Valentine leukocidin, and the multidrug resistance plasmid pSK41. $J$ Clin Microbiol 2003;41:3017-3021.

30. Melles DC, Gorkink RF, Boelens HA, et al. Natural population dynamics and expansion of pathogenic clones of Staphylococcus aureus. I Clin Invest 2004;114:1732-1740.

31. Weidenmaier C, Kokai-Kun JF, Kristian SA, et al. Role of teichoic acids in Staphylococcus aureus nasal colonization: a major risk factor in nosocomial infections. Nat Med 2004;10:243-245. 